\title{
Das leitende Element in den Muskelfasern von Ascaris.
}

Von

Professor Dr. Stefan Apáthy in Kolozsvár.

Hierzu Tafel XXXVI.

Ich habe den Bau der Längsmuskelfasern von Ascaris lumbricoides kürzlich in einer längeren Abhandlung in der Zeitschrift für wissenschaftliche Mikroskopie ${ }^{1}$ ) ausführlich beschrieben. Da ich in jener Arbeit, in welcher ich das Hauptgewicht auf die Schilderung der nach meiner Ansicht bei ähnlichen Untersuchungen am besten anzuwendenden Mikrotechnik legte, und die mit dieser Technik wahrgenommenen morphologischen Feinheiten hauptsächlich bloss zur Illustration der Technik selbst dienen wollten, so glaubte ich meiner Beschreibung nicht nothwendigerweise auch Abbildungen alle $\mathrm{r}$ besprochenen Verbältnisse beigeben zu müssen. Auch hatte und habe ich die Absicht, das Nervensystem von Ascaris in einer besonderen Schrift eingehender zu behandeln. Ein wesentlicher Theil der dazu nöthigen Abbildungen war schon damals fertig, namentlich Alles, was sich auf den engeren Zusammenhang des leitenden Elementes, nach meiner Ueberzeugrung der leitenden Primitivfibrillen, mit den Muskelfasern bezieht. Einen wichtigen Theil jener Abbildungen habe ich bereits im Juli des vergangenen Jahres dem Redakteur der Zeitschrift für wissenschaftliche Mikroskopie, Herrn D. W. J. Behrens vorgezeigt.

Anderweitige Verpflichtungen zwingen mich die Veröffentlichung meiner Untersuchungen über das Nervensystem von Ascaris aufzuschieben. Ich weiss noch nicht, anf wie lange Zeit dieses geschehen muss; deshalb scheint es mir geboten wenigstens den Antheil, welchen die leitenden Formelemente an dem Auf-

1) Bd. X, Heft 1 (1893), p. 36-73 mit Tafel III, und Heft 3 desselben Bandes, p. 319-361. 
bau resp. in der Versorgung der Längsmuskelfasern von Ascaris haben, in Zusammenhang mit meiner oben citirten Arbeit durch einige Abbildungen zu veranschaulichen.

Eine besondere Veranlassung giebt mir dazu ein Artikel von $\mathrm{Rohde}$, welcher jüngst im Zoologischen Anzeiger ${ }^{1}$ ) erschienen ist, dessen betreffende Nummer mir eben heute (am 16. Februar) in die Hände gekommen ist.

Meine Entdeckung der leitenden Primitivfibrillen, welche in die Ascarismuskelfasern eintreten, diese z um $\mathrm{Tb}$ eil durchsetzen und sich in die Subeuticularschichte fortsetzen, bezeichnet R ohde als widersinnig. Für sein Urtheil hat er nicht weniger als vier Gründe. Wenn die Gewichtigkeit derselben so gross wäre, wie ihre Zahl, so wäre ich vollkommen geschlagen. Ja er hat sogar noch ein fünftes Argument: er ergeht sich nämlich in persönlichen Angriffen. Für letztere habe ich kein Gegenargument; dazu jedoch, dass es mir gelingen wird die ersteren etwas abzuschwächen, habe ich noch Hoffnung.

Das erste Argument basirt sich auf "theoretisch physiologische Erwägungen". Auf was sich aber letztere bei Rohde basiren, wollen wir gleich sehen! Zunächst wird aus meiner Schilderung der leitenden Primitivfibrillen in ibrem Zusammenhang mit der Muskelfaser eine vollkommen unberechtigte Consequenz gezogen. „Die Subcuticularfibrillen können doch nur als sensibel aufgefasst werden. Da nun A páthy angiebt, dass sie die direkte Fortsetzung der nervösen Fibrillen der Muskelmarksubstanz sind, so ständen wir zunächst vor dem physiologischen Curiosum, dass eine und dieselbe Fibrille theils centrifu-

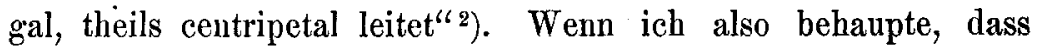
ein Nervenstamm sowohl Muskeln, als auch Sinnesorgane versieht, d. L. ein Nerv gemischten Charakters ist, so folgt daraus, dass eine und dieselbe Nervenfaser oder Fibrille theils centrifugal, theils centripetal leitet! Ebenso gemischten Charakters sind die Bündel von leitenden Primitivfibrillen, welche - meist durch Vermittlung der sogenannten Markbeutelfortsätze oder Muskelquerfortsätze - in die Muskelfaser eintreten. Ein Theil der-

1) Rohde, E., Apáthy als Reformator der Muskel- und Nervenlehre. Zoologischer Anzeiger No. 439 (1894), p. 38-47.

2) Rohde, l. c. p. 41.

Arehiv f. mikrosk. Anat. Bd. 43 
selben bleibt in der Muskelfaser, indem sie sich in dem Zwischenraum zwischen je zwei contractilen Leisten, wohin sie in radiärer Richtung einzeln eindringen, umbiegen und dort eine longitudinale Richtung annehmen, in welcher sie als je eine scharfe Linie, nicht aberalsein FilzwerkfeinerSpongioplasmafäserchen, oft weit zu verfolgen sind (s. Figur 8). Der andere Theil der Primitivfibrillen setzt die Rinde der Muskelfaser in radiärer Richtung, ebenfalls einzeln zwischen je zwei contractilen Leisten, durch und tritt in die Subcuticula ein. Dieses Verhalten habe ich in meiner Arbeit ausfïhrlich beschrieben, mit besonderer Betonung dessen, dass jene Fibrillen keineswegs eine fadenartige Verflechtung von kleineren Füserchen sind, wie es dem Spongioplasma R o h d e's entspricht, sondern ganz continuirliche, scharf gezeichnete wirkliche Fibrillen mit - abgesehen von kleinen, im mikroskopischen Bild zum Theil bloss vorgetäuschten Verdickungen, Varicositäten - vollkommen $g$ lat te n Contouren, wie es besonders in Figur 6 und 7 des vorliegenden Aufsatzes deutlich sichtbar ist. Sie unterscheiden sich in diesen Zeichnungen eben so gut wie im Präparat durch ihre die aller übrigen Gebilde übertreffende Dunkelheit und Seh ärfe, sodass sie mit anderen etwa faserig erscheinenden Constituenten des mikroskopisehen Bildes in den nach meiner im erwähnten Aufsatz genau beschriebenen Goldmethode gemachten Präparaten absolut nicht $\mathrm{zu}$ verwechseln sind ${ }^{1}$ ). Das alles habe ich schon damals betont. Auch habe ich dargelegt, dass diese Fibrillen in ihrer Continuität, auf weite Strecken verfolgbar, a u ch zu i soli r e n sind.

Nun kann von den Primitivfibrillen, die, in ein und dasselbe Bündel vereinigt, in die Muskelfaser eintreten, derjenige Theil, welcher in der Muskelfasser verbleibt, oder aus ihr wenigstens nicht nachweisbar heraustritt, als m o t o ri sc h aufgefasst werden; derjenige Theil dagegen, welcher durch die Rinde in die Subcuticularschicht heraustritt, ist wahrscheinlich als sensorisch zu betrachten. Eigenthtimlich ist es, aber es ist eben so, dass die sensorischen Fibrillen ihren Weg von der Subeuticula in die betreffende Medianlinie, wo sie zum Nervencentrum geleitet werden, oder auch direkt zum Schlundring - falls sie nicht bereits

1) Apathy, l. c. p. $348-350$. 
früher ihr Ziel in Ganglienzellen der Medianlinie selbst erreichen - durch die Muskelfasern nehmen und nicht den Zwischenraum zwischen je zwei Muskelfasern dazu bentitzen.

Logisch kann man aus meiner Schilderung nur diese Consequenzen ziehen. Entweder hat sie Rohde nicht ganz durchgelesen oder er hat sie nicht verstanden. Es giebt jedoch auch eine dritte Alternative; an diese will ich nicht denken, obwohl er mir noch eine ganze Anzahl anderer Behauptungen imputirt, mit denen ich nichts zu thun habe. "Die Muskelzelle besteht also zum grössten Theil aus nervösen Elementen $\left.{ }^{1}\right)$ !" So etwas habe ich nie behauptet. Niemand wird es glauben, dass ich dieser Meinung bin, wer meine Arbeit mit unparteiischem Sinne liest. Ich betone es wiederholt, dass der grösste Theil der Muskelfasern aus dem Muskelplasma mit sehr viel Zellsaft besteht. Das Muskelplasma schildere ich an der Hand mehrerer Methoden ganz eingehend, und zwar in seinen verschiedenen Zuständen, wo es schaumig-wabig, resp. spongiös aussieht (s. Figur 6 und besonders 9) und wo es den Anschein hat, als bestände es beinahe nur aus einer dichten Granulamenge (s. Figur 7). In dieses oft spongiöse Muskelplasma eingebettet verlaufen die von mir entdeckten und durch meine Methode so dentlich darzustellenden Fibrillen, indem sie sich mehrfach verzweigen, resp. ans dickeren Bündeln in dünnere oder in einzelne Fibrillen auflösen; wo sic in grösserer Anzahl vorhanden sind (Figur 6), verflechten sie sich auch. Dieses Geflecht ist aber etwas ganz Anderes als das Spongioplasma Rohde's. Was ich gefunden habe, sagt $\mathrm{Rohde}$, ist nicht neu, sondern von ihm bereits beschrieben. Offenbar vermochte er sich gar keine Vorstellung von dem von mir beschriebenen Fibrillensystem zu machen, sonst würde er dies kaum behaupten können. Wenn man seinc Zeichnungen mit den meinigen vergleicht, so wird man sich sofort überzeugen, dass ihm seine Präparate gar keine Ahnung von diesen Verhältnissen geben konnten.

Ich veröffentliche nun meine Abbildungen, welche mit den besten optischen Hilfsmitteln, immer mit dem $\mathrm{Abbe}$ 'schen Zeichenapparat verfertigt worden sind. Sie sind, wo nicht Anderes angegeben, die getreneste Wiedergabe des betreffenden mikroskopischen

1) Rohde, I. e. p. 41. 
Bildes bis auf jedes Strichlein, bis auf jeden Punkt. Ich habe meine Zeichnungen, bei mangelnder Zeit, lieber nicht ausgefübrt, d. h. nur stellenweise Alles eingezeichnet, als dass ich an ihnen etwas schematisirt hätte. So ist z. B. in Figur 6 bloss die rechte Muskelzelle ganz ausgeführt, in die linke sind im Mark bloss leitende Fibrillen, welche im Bilde, wie gesagt, sehr scharf differenzirt waren, eingezeichnet. In Figur 9 ist dagegen bloss das Mark einer Partie der Muskelzelle ausführlich eingezeichnet; die contractile Rinde bloss angedeutet. In Figur 7 ist das eine Granulastructur zeigende Mark bloss an zwei kleinen Stellen eingezeichnet; die Rinde ist bloss in dem unteren Theil der Figur ausgeführt; dagegen ist Alles, was von leitenden Fibrillen in dem betreffenden Querschnitt der Muskelfaser zu sehen war, genau wiedergegeben, u. s. w. Vieles ist in den Figuren nicht gezeichnet, was aber da ist, ist so getreu, wie es nur Zeichenapparat und eigenes Können erlaubten, dargestellt. Dass jedoch aus diesen Figuren, wenn man sie mit denen von $\mathrm{R}_{0} \mathrm{hd}$ e vergleicht, „die Gleichheit der von uns beschriebenen Fibrillen ohne Weiteres einleuchten wird ${ }^{\text {}}$, glaube ich nicht.

Seine Methode, und noch etwas Anderes, haben es Rohde nicht gestattet, in dem Mark der Muskelfasern von Ascaris mehr als bloss sein Spongioplasma wahrzunehmen. Meine Methoden bewirken dagegen eine weitere Differenzirung sowohl des Markes, als anch der Markbeutelfortsätze, der Subcuticula, der Nerven und des Schlundringes. Neben dem und in $\mathrm{dem}$, was er als Spongioplasma zu bezeichnen vermag, sind dort auch Fibrillen, continuirliche Fibrillen $\mathrm{g}$ an $\mathrm{z}$ anderer Art vorhanden, welche ich zuerst entdeckt habe. $O b$ ich berechtigt war, diese als leitend anzunehmen, darauf komme ich weiter unten noch zurïck. Nun hat Rohde mit den von mir so ausführlich beschriebenen Methoden nicht nachuntersuchti); also könnte ich ihm einfach sagen, dassergar nicht berechtigt ist, über meine Angabenein Urtheil za fällen, mag er seine eigenen Präparate, so lange er

1) Der zweite, hier hauptsächlich in Betracht kommende Theil meiner Arbeit ist am 7. November 1893 erschienen, Rohde's Entgegnung am 22. November 1893 dem Zoologischen Anzeiger eingegangen. Offenbar wäre die Zeit zum Nachuntersuchen zu kurz gewesen. Auch erwähnt Rohde von einem solchen gar nichts. 
will, wiederholt bewundern, zumal seine allgemeinen "theoretischphysiologischen Erwägungen" soleher zweifelhaften Natur sind, wie es gleich des weiteren gezeigt wird.

„Bei der ganz aussergewöhnlichen Ausbildung nervöser Fibrillen in Musculatur und Subcuticula müssten wir ferner einerseits auf eine sehr grosse Beweglichkeit der Thiere, andererseits auf einen ganz bedeutenden Reichthum von Sinnesorganen schliessen. Bekanntlich zeigt die Wirklichkeit gerade das Gegentheil. Die Thiere sind äusserst träg und sehr arm an Sinnesorganen, welche hier als Papillen auftreten " $\left.{ }^{1}\right)$. Die von mir entdeckten Fibrillen - ich spreche n i c h t vom Spongioplasma, welches wirklich nichts mit der Nervenleitung zu thun hat! - können also nicht nervöser Natur sein, weil die Thiere zu wenig beweglich sind. Gut! Die anch von $\mathrm{R}$ ohde und vor ihm von vielen anderen Forschern beschriebenen Gebilde, welche als Muskelfasern bezeichnet werden, namentlich aber die grosse Menge contractiler "Säulchen", welche auch Rohde beschreibt, das Alles hat mit der Muskelthätigkeit nichts zu thun, sie können keine Muskeln sein, weil das Thier zu einer so grossen Menge Musculatur viel zu träge und unbeweglich is t. Ist die Menge der leitenden Fibrillen mit der B eweg li c hke it in einem so einfachen direkten Verhältniss, wie Rohde meint, um wie Vieles mehr muss das zwischen contractiler Substanz und Beweglichkeit der Fall sein? Wenn Rohde aus diesem, nach seiner Folgerung zwingendem, Grunde öffentlich erklärt, dass die bisher von der ganzen Welt für Muskelfasern gehaltenen Gebilde bei Ascaris keine Muskelfasern sind, dass er sich hierin auch geirrt hat, so bin ich ebenfalls bereit zu erklären, dass das von mir entdeckte System von Fibrillen nicht leitender $\mathrm{Natur}$ ist.

Wo die Menge der Muskelfasern so ausserordentlich gross sein kann, ohne dadurch eine grosse Beweglichkeit zu verursachen, wie bei Ascaris, dort kann auch die Menge der Nervenprimitivfibrillen eine auf den ersten Blick befremdend grosse sein, ohne deshalb eine grosse Empfindlichkeit des Thieres nothwendig zu machen: wo die contractile Substanz massenhaft entwickelt ist, dort kann es ebensogut auch die leitende Substanz

1) Rohde, 1. c. p. 42. 
sein. Und andrerseits glaube ich nach dem, was ich von Leben und Lebenden weiss, dass, je weniger ein Thier mit Sinneswerkzeugen ausgestattet ist, eine um so grössere Ausdehnung muss die, wenn auch in einem geringen Grade, aber doch empfindliche Körperoberfläche haben, und das Thier muss eine relativ uщ so grössere Menge leitender Buthnen und leitender Substanz überhaupt besitzen, damit es, trotz der so schlechten Ausnützung der Nervenleitung, doch irgendwelche äussere Eindrücke wahrnehmen kann. Auch muss in solchen Fällen, namentlich bei Ascaris, wohl die Quantität der leitenden Substanz für die. noch wenig verfeinerte $Q u$ alit ät entschädigen. $\mathrm{Mehr} S$ inneso rgane würden Ascaris dazu befähigen, we ni ger le i te nde $\mathrm{B}$ a h n en und weniger leitende Substanz bes ser a us u n ü tzen und somit wäre die grosse Menge, welche bei ihr nachzuweisen ist, erst recht befremdend, weil sie unnöthig wäre.

Die Schilderung, nach ihm bloss die "Deutung" meiner Fibrillen, sucht $\mathrm{R}$ oh d e unter Anderem auch dadurch ad absurdum zu leiten, dass er aus ihr die Consequenz zieht: „Die Subcuticula hat also einen vorwiegend nervösen Charakter!" ${ }^{1}$ ). Jawohl, das hat sie: keinen vor w i e ge nd nervösen aber a u ch e in en n er vö s e n Charakter, indem sie die äusserste zellige Körperschichte ist, in welcher die periphere Ausbreitung einer grossen Anzahl leitender Fibrillen stattfindet.

Glaubt denn aber $\mathrm{R}$ o h de, dass ich die Zellkerne, welche in die Grundmasse der Subcuticula, stellenweise sehr dicht, eingestreut sind, nicht kenne? Zu diesen Zellkernen gehört auch je ein Zellkörper, wenn auch die Grenzen und Contouren derselben in der homogenen Zwischensubstanz, in welche sie eingebettet sind, verschwinden. Nun ist der Zellkörper eine protoplasmatische Substanz und somit vollkommen geeignet, um im mikroskopischen Bild eine Structur aufzuweisen, welche $R$ o h d e als spongiös bezeichnen mag. Auch die an und für sich hyaline Grundsubstanz ist derart, dass sie beim Erstarren in Folge der angewandten Fixirungsmittel, resp. bei der weiteren Behandlung eine verworren fibrilläre Structur annehmen kann. Das sind aber bloss scheinbare oder nicht präformirte Fibrillen, welche der Subcuticula das Aussehen eines Filzwerkes aus feinen, kurzen, regellos verflochtenen Fäserchen

1) Rohde, l. c. p. 41 . 
geben können. Dieses für mich jetzt unwesentliche Filzwerk ist nun von den scharf gezeichneten, genau zu verfolgenden Fibrillen durehzogen; die dickeren unter ihnen werden von dünneren Primitivfibrillen zusammengesetzt, welche, wie bereits offters betont wurde, einzeln durch die Zwischenleisten, in denen sie je eine radiäre Mittellinie bildeten, aus den Muskelfasern heraustreten. Dieses Verhältniss ist in Figur 6 sehr deutlich zu sehen. Die Grundsubstanz der Subcuticula geht in die Interstitialmembran, welche die Muskelfasern resp. deren Markbeutel und Markbeutelfortsätze gegen die Körperhöhle zu bedeckend, sich zwischen je zwei Muskelfasern radiär hineinschiebt, wie es besonders Figur 10 zeigt, ununterbrochen über.

Doch will ich hier Schilderungen, welche sich in meiner ausführlichen Arbeit finden, nicht wiederholen. Die Subcuticula von Ascaris entspricht gleichzeitig der Epithelschichte und dem subepithelialen Bindegewebe der höheren Würmer. Ebenso wie dort das Epithel und seine direkteren Abkömmlinge, die eventuell tief in den Körper hineingesunkenen einzelligen Drüsen, hat hier die Subcuticula in erster Linie für den Schutz des Körpers durch Absonderung der dicken Cuticula zu sorgen. Nach der Mehrzahl seiner Zellen, welche einzellige Drüsen sind oder zu solchen werden sollen, ist das Epithel von Lumbricus eine in erster Linie absondernde Schichte, und doch hat diesel Umstand Lenhossék und Retzius nicht verhindert, das Eintreten von sehr zahlreichen Nervenfibrillen in das Epithel nachzuweisen. Bereits bat sich sogar ein Leuckart für die Annahme Len hosséks, dass das Epithel von Lumbricus und wahrscheinlich vieler anderer Thiere auch nervöser Natur ist, ausgesprochen. Warum soll die Subcuticula von Ascaris, welche gleichzeitig auch dem Epithel von Lumbricus entspricht, nicht auch unter Anderem nervöser Natur sein? Le nh o s sék würde unter den Zellen der Subcuticula vielleicht sensorische Nervenzellen suchen. I ch meinerseits will nur so viel nachgewiesen haben, dass die sensorische Endausbreitung des Nervensystems von Ascaris in der ganzen Subcuticula stattfindet. Ist das bei dem heutigen Stande unseres Wissens über das Nervensystem der niederen Thiere ein Absurdum? Ich schmeichle mir, dass meine Angaben für die Mehrzahl der gegenwärtigen Nervenforscher 
eher ein willkommener Beitrag zu unseren Kenntnissen sein werden.

Noch einen Einwand, welcher in die Kathegorie der "theoretisch-physiologischen Erwägungen" Rohde's gehört, will ich erwähnen. Das betreffende Argument kann in der folgenden Weise zusammengefasst werden: Der "Achsencylinder" der Nervenfaser, welcher je eine Papille von Ascaris versorgt, zieht „unter Verjüngung bis an die Oberfläche der Cuticula“. Die Subcuticularfasern treten aber „niemals über die Papille hinaus in die Cuticula", folglich können sie keine Nervenfibrillen sein $\left.^{1}\right)$. Dagegen frage ich einfach, ob denn die Nerven im Körperoberflächenepithel von Lumbricus in die Cuticula eintreten; ob denn die Nervenfasern der Epidermis der Wirbelthiere a uf die Oberfläche derselben hinaustreten? Sind denn deshalb jene Nerven keine Nerven? Was haben die Nerven, ausgenommen bei ganz speciellen Endigungsweisen, in der Cuticula, welche ja bei den meisten Würmern wiederholt abgeworfen wird, zu thun?

Das zwe ite Argument Rohde's lautet folgenderweise. Auf die Frage, was mich dazn berechtigt jene Fibrillen für nervös zu erklären, findet sich bei mir angeblich bloss die „Erklärung, dass sie gerade so aussehen wie die von mir "bei Hirudineen, Chaetopoden, Crustaceen etc. für nervös betrachteten Elemente." Nun sei es aber evident, dass es ein Absurdum ist, Subeuticularfibrillen als leitend anzusehen, und so habe ich meiner nganzen Nervenreformationstheorie dadurch, dass ich sie auf die Nematoden ausdehnte, selbst den Todesstoss gegeben " 2 ). Mit anderen Worten: die Fibrillen sind nicht nervös, weil sie nicht nervös sind! Ich weiss nicht, was auf eine solche Beweisführung zu antworten ist.

Rohde irrt sich sehr, wenn er glaubt, dass er es irgendwie nachgewiesen hat, dass die Primitivfibrillen, welehe ich besonders bei Hirudineen genan geschildert habe, nicht leitender Natur und nicht das eigentlich Wesentliche der Nervenfasern sind. Meine Methylenblaumethode ${ }^{3}$ ) lieferte mir ganz schlagende

1) l. c. p. 42 .

2) 1. c. p. 43 .

3) Apathy, St., Erfahrungen in der Behandlung des Nervensystems für histologische Zwecke. I. Mittheilung: Methylenblau. Zeitschrift f. wissensch. Mikroskopie, Bd. IX (1892), p. 15-37. 
Beweise für die Richtigkeit meiner Auffassung und bestätigte das, was schon an meinen früheren Goldchloridpräparaten so klar gewesen ist ${ }^{1}$ ); nun kommt jene Goldchloridmethode dazu, welche ich in meiner Ascarisarbeit empfohlen habe, welche Alles nochmals bestätigt, ja sogar Vieles, da hier feine Schnittreihen behandelt werden können, noch deutlicher hervorhebt (s. die Figuren $3-10$ ).

Uebrigens habe ich bloss erwähnt, dass die betreffenden Fibrillen bei Ascaris genau dieselben Reactionen zeigen, wie jene Primitivfibrillen, von welchen ich bei Hirudineen, Lumbricus etc. bereits nachgewiesen habe, dass sie die leitende Substanz sind. Rohde war nicht im Stande, sie bei den Hirudineen zu sehen; er sah bloss Spongioplasma; auch bei Ascaris kann er sie nicht sehen, auch hier sieht er bloss sein Spongioplasma. Das aber finde ich natürlich, dass sein Spongioplasma hier, wie dort gleich aussieht; ebenso natürlich ist es andrerseits, dass die von mir nachgewiesenen leitenden Primitivfibrillen bei den verschiedenen Objecten gleich aussehen. Ausser dieser Gleichheit habe ich noch ganz andere Gründe aufgefuhrt und das Hauptgewicht auf diese gelegt: ich habe die leitenden Primitivfibrillen von der Subcuticula einerseits in die Mediannerven, andererseits in die Muskelfasern, von dort in die Markbeutelfortsätze, weiter in die Mediannerven oder in den Schlundring, resp. bis zu peripherischen Ganglienzellen, in ihrer ununterbrochenen Individualität - in grössere Bündel, bezüglicherweise dickere Fibrillen vereinigt - verfolgt (s. Figur 3, 4 und 5).

Es entspricht also nicht der Wahrheit, dass mein alleiniger Grund, die Fibrillen für nervös zu erklären, ihr Aussehen gewesen ist. Auch ist Alles, was er über die von mir aufgestellte Analogie des Baues von Muskelfaser und Nervenfaser erwähnt, ganz verdreht. Er könnte es ganz gut wissen, wenn er meinen Aufsatz: Contractile und leitende Primitivfibrillen ${ }^{2}$ ), den er citirt, auch gelesen hat, dass ich ebenso, wie von Muskelfasern zweierlei Typen von Nervenfasern (Nervenspindeln) unterscheide, nämlich den bündelförmigen und den röhrenförmigen

1) Apathy, St., Contractile und leitende Primitivfibrillen. Mittheilungen a. d. zoolog. Station zu Neapel, Bd. X 1892), p. 355-375.

2) S. die vorige Anmerkung. 
Typus. Aus der Darstellung der Nerven von Ascaris, welche ich auf Seite 357 meiner Ascarisarbeit, obwohl ich auf die Schilderung des Nervensystems nicht cingehen wollte, gegeben habe, sowohl als anch aus dem auf Seite 358 Gesagten, ist es ganz deutlich, dass ich die Nerven von Ascaris ebenso wie zum Theil sogar die der Hirudineen, welche zu einem gemischten Typus gehören, als bü $\mathrm{ndel}$ fö $\mathrm{rmig}$ betrachte: in ihnen verlaufen die leitenden Primitivfibrillen, gerade wie in den verjüngten Strecken der Markbeutelfortsätze, in $\mathrm{B} u ̈ n d e l$ vereinigt und $\mathrm{nicht}$ in der Wand einer Röhre (s. Figur 5). Also weise ich folgenden Satz Rohde's als incorrect zurück: „Dem entsprechend hält er auch bei der Nervenfaser von Ascaris den hellen feinfibrillären, oben als Achsencylinder bezeichneten Inhalt (Fig. 2) nur für eine nicht nervöse Marksubstanz und die die Scheide bildenden Subcuticularfibrillen für die leitenden Elemente" ${ }^{1}$ ). Meine Arbeiten über die Nervenfasern der Mollusken und der Crustaceen sind wohl nicht so ausfübrlich, auf alle Einzelheiten eingehend, wie über die der Hirudineen, Lumbricus und Ascaris, im Wesentlichen wird aber das Wenige, was ich über jene bereits mitgetheilt habe, auch von den künftigen diesbezüglichen Mittheilungen $R o b d e$ 's wohl nicht viel zu fürchten haben. Vielleicht wird sich Rohde bis dann sogar die Technik, welche eine deutliche Differenzirung der leitenden Primitivfibrillen gestattet, aneignen: bisher hat er sie nie gesehen.

Das dritte Argument $\mathrm{R}$ ohd e's lautet etwa folgendermaassen: Seine Präparate sind nach sehr guten, bewährten Methoden verfertigt, auch sind sie sehr schön; die mikroskopischen Bilder, welche sie von der Beschaffenheit unseres Objectes geben, sind sehr scharf, folglich existirt in dem Object nichts weiter, als was $R_{0} h d e$ an ihnen zu sehen im Stande ist, folglich existiren die von mir prätendirten leitenden Primitivfibrillen nicht resp. ist das von mir bei Ascaris beschriebene Fibrillensystem nichts weiter, als das Spongioplasma Rohde's, welches nicht das Leitende sein kann.

Was nun zuerst die Methoden Rohde's anbelangt, so habe ich es in meiner Ascarisarbeit zur Genïge anseinandergesetzt, war um ich solche zur Untersuchung der uns interessiren-

1) Rohde, l. c. p. 43 . 
den Verhältnisse für unzulänglich halte. Ich betonte es wiederholt, dass ich bei Ascaris ausser den eingehender beschriebenen Methoden auch so manche andere Fixirungen und Färbungen, Isolationsmethoden und Imprägnirungen angewandt habe, unter diesen auch alles von $R$ ohde Empfohlene. Es war mir ja, aus Gründen, welche keinen directen Bezug auf das in der Ascarisarbeit Erstrebte haben, hauptsächlich daran gelegen, di e Resultate verschiedenster Methoden der modernen Mikrotechnik bei einem und demselben Object genau zu vergleichen. Wenn ich die Methoden Rohde's für unzulänglich erklärte, so that ich dies deshalb, weil ich die seinigen ja nachgeprüft habe und weil andere Methoden einerseits das bereits mit der Technik Rohde's Sichtbare noch schärfer hervortreten lassen und hauptsächlich v or w u r f s f r e i e r e Schlüsseaufdienatürliche Beschaffenheit unseres 0 bjectes gewähren, andrerseits aber Manches, was in den Rohde'sehen Präparaten absolut nicht sicbtbar sein kann oder wenigstens nur sehr schwer erkenntlich ist, sicherund deutlich differenziren, so namentlich die leitenden Primitivfibrillen.

Dass $R \circ \mathrm{h}$ d e seine eigenen Präparate schön findet, ist eben Geschmacksache, und, nach seinen Resultaten zu urtheilen, auch ein Zeichen gewisser Genügsamkeit. War er ja vollkommen überzeugt, und ist es wohl auch noch heute, dass die Präparate, hauptsächlich Paraffinserien, anf welche sich seine Arbeit über das Nervensystem der Hirudineen gründete, sehr schön und vollkommen überzeugend sind. $\mathrm{Er}$ war so freundlich sie mir und einigen anderen Fachgenossen in Neapel zu demonstriren, in der Hoffnung mich und jene anderen Herren von der Richtigkeit seines Standpunktes, gegenüber dem meinigen, zu überzeugen. Nun waren ja diese Präparate sehr nett hergestellt, die Schnitte sehr dünn und als solche tadellos; das, was man unter dem Mikroskop an ihnen sehen konnte, erschien sehr rein, deutlich und scharf gezeichnet, ebenso wie die Abbildungen, welche Rohde von ihnen verfertigte. Leider war an ihnen das Wesentliche undifferenzirt und deshalb das unsichtbar, was bereits an meinen damaligen, noch mehr aber an meinen neueren, namentlich Methylenblau- und Goldchloridschnittserienpräparaten so deutlich zu Tage tritt: ich meine die Existenz, die 
Beschaffenheit und den Verlanf der leitenden Primitivfibrillen. Das Sichtbare dagegen forderte ganz dringend jenes granum salis, welches allein den Forscher befähigt, aus den unvermeidlichen Kunstproducten seiner Präparate richtige Schlissse auf die natürliche Beschaffenheit seines Objectes zu ziehen. Von den Urtheilen jener Fachgenossen darf ich wohl so viel erwähnen, dass sie mit mir darin vollkommen übereinstimmen, dass das Kanstproduct in den uns demonstrirten Präparaten etwas iiber das Unvermeidliche hinansgegangen war. Jedenfalls bin ich Rohde für seine liebenswiirdige Demonstration zu grossem Dank verpflichtet, da er mich durch diese in der Ueberzeugung von der Richtigkeit meiner e ige n en Anschaung der seinigen g e $g$ e $n \ddot{u}$ be $r$ ganz wesentlich verstärkt hat.

Was nun meine armen Muskelpräparate betrifft, welche ich ihm damals demonstrirte, so sind diese freilich derart, dass ich mir nach ihnen "nichts träumen" lasse von der Schärfe, mit welcher in der Muskelzellrinde „die contractilen Leisten nach Osmiumsäure-Fixirung auf Schnitten" bei Ascaris hervortreten. Es ist wirklich ein Wunder, dass sie es doch vermochten, Rohde von der vollkommenen Unrichtigkeit seiner früheren Darstellungen der Muskelfasern versehiedener Thiere zu überzeugen und ibn, was die Beschaffenheit der contractilen Substanz betrifft, vollkommen zu meiner Anschauung zu bekehren, - was er ja in seiner citirten Entgegnung selbst zugiebt. Zu meiner Entschuldigung soll dienen, erstens, dass ich wohl schon damals (im Sommer 1891) über bessere Präparate verfügte, als welche ich in Neapel, auf einer Reise, bei mir hatte; zweitens, dass die Beschaffenheit der contractilen Rinde aus alternirenden contractilen Leisten und Zwischenleisten sowohl als auch deren Natur bei Ascaris unvergleichlich auffälliger und leichter zu demonstriren ist, als bei den Hirudineen; und drittens, dass seit jener Zeit auch ich um einige kleine Schritte in der Technik, meinen Gegenstand zu bewältigen, vorwärts gekommen bin.

Alles das ist hier jedoch Nebensache! Die Hauptsache ist, dass, auf Grund von in Betreff gewisser Verbältnisse ne gativen Resultaten, welche er mit sonst noch so guten Methoden erhalten hat, niemand berechtigt ist die positiven Resultate, welche nach ganz anderen Methoden erzielt worden sind, anzuzweifeln. Seine Pflicht ist mit denselben Methoden nachzuuntersuchen, und wenn 
er, trotz der skrupulösesten Sorgfalt in der Ausführung der vorgeschriebenen Methode an demselben Objekt und trotz sehr oft wiederholter Versuche, dennoch keine positiven Resultate bekommt, - nun dann kann er auch nicht mebr, als die Methode für unsicher zu erklären, und die betreffenden angezweifelten Verhältnisse als ein Ereigniss im Präparat, welches nicht ohneWeiteres auf die natürliche Beschaffenheit des Objectes bezogen werden soll, zu bezeichnen. Bekommt er dagegen positive Resultate, so mag er dieselben nach ihrer Beweiskraft analysiren oder, wenn er es kaun, zu Gunsten seines eigenen Standpunktes erklären. Kann er dies nicht, so muss er nachgeben. Die Präparate z. B., welche man nach Chromsäurefixirung und Härtung und Tinktion im alten ammoniakalischen Carmin von Gehirn und Rückenmark der Wirbelthiere bekommt, können in ihrer Art sehr schön sein und lassen von den hochwichtigen Verhältnissen, welche uns erst die Golgi'sche Methode enthüllt hat, doch gar nichts ahnen. Wäre es richtig immer nur auf den Untersuchungsresultaten, welche man an den alten Präparaten gewonnen hat, herumzareiten und deshalb von mit anderen Methoden gewonnenen Resultaten nichts wissen zu wollen? Etwas ganz ähnliches thut mir gegenüber Rohde, über dessen histologische Technik, was die Herstellung der Nervenpräparate betrifft, die verschiedenen Goldmethoden, die Chromsilbermethoden, die Methylenblaumethoden etc. spurlos hinweggeflogen sind, um von meinen eigenen Verfahren, welchen ich meine von ihm bestrittenen Angaben verdanke, gar nicht zu reden. Nur so ist es zu verstehen, dass er so hartnäckig an der unglücklichen Hyaloplasmatheorie der Nervenleitung festhält, wo doch unsere heutige Kenntniss der Vertheilung und der Endigungsweise der leitenden Bahnen (- vielfach nicht in den Zellen, sondern zwischen den innervirten Zellen -) dieselbe geradezu unmöglich macht.

Gehen wir aber endlich zum vierten und letzten Argument Rohde's über! Dieses ist kein geringeres, als das Constatiren der Thatsache, dass er seine Schilderungen mit vielen, mühevollen Abbildungen erläutert, wogegen ich das von mir Behauptete entweder nur mit wenigen, ungenitgenden Zeichnungen oder überhaupt nicht illustrirte. Folglich verdient seine Darstellung volles Vertrauen; die meinige erweckt dagegen Misstranen. - Warum ich in meiner Ascarisarbeit das, was sich auf das Leitende in den 
Muskelfasern bezieht, nicht mit Abbildungen begleitet habe, ist dort deutlich mitgetheilt und wurde bereits auch hier erwähnt. In meinem hier ebenfalls citirten Artikel über contractile und leitende Primitivfibrillen ist das im Text Auseinandergesetzte durch Abbildungen, wie ich glaube, in genügender, weil überzengender Weise erläutert. Ich hätte wohl auch mehr geben können; allein ich spare meine Zeichnungen, welche sich auf Hirudineen beziehen, für eine seit langen Jahren verbreitete grössere Monographie auf: auch das habe ich bereits bei verschiedener Gelegenheit erklärt. Und was für Abbildungen und wie viele ich, besonders von dem feineren Bau des Nervensystems besitze, darüber könnte die Direction der zoologischen Station zu Neapel, auf welcher die Verlagskosten meiner Monographie lasten, etwas reden.

Dagegen steht nichts im Wege, dass ich die Rolle des leitenden Elements in der Muskelfaser und in der Subcuticula von Ascaris an der Hand einiger Abbildungen hiermit noch besser erläutere. Ich gebe nur das, was ich zu diesem Zweck fiir nothwendig erachte.

Fig. 1 und 2 sind nach Quetschpräparaten in 50procentigem Glycerin gezeichnet und sollen die Reaction der frischen, lebenden Muskelrinde nach der Goldchloridameisensäuremethode, welche auf Seite 334 und 335 meiner citirten Ascarisarbeit beschrieben ist, veranschaulichen. Die contractile Rinde der Muskelfuser, und besonders die contractilen Leisten selbst sind hier stark gequollen. (Vergleiche letztere in Fig. 2 bei 1500 facher und in Fig. 6 bei 2000 facher Vergrösserung.) Die contractilen Leisten cl sind im Präparat sehr blass hortensia roth, kaum gefärbt, wogegen die Zwischenleisten zl stark tingirt, kirschroth erscheinen, mit meist dunklen, beinahe schwarzen, eingelagerten Körnchen, welche in Fig. 1 nur stellenweise eingetragen, dagegen in Fig. 2 genau eingezeichnet sind.

Figur 1 ist ein Stïckchen der contractilen Rinde mit vertikal auf das Gesichtsfeld stehenden contractilen Leisten, von aussen betrachtet, bei bloss 400 facher Vergrösserung. Sie zeigt besonders zwei Thatsachen deutlich: erstens dass die contractilen Leisten bloss gruppenweise ganz parallel zu einander verlaufen, die einzelnen Gruppen dagegen, innerhalb gewisser Grenzen, verschiedene Richtungen annehmen kömnen; zweitens dass die contractilen Leisten verschieden lang sind und es auch ganz kurze, 
inzwischen eingekeilte, wie bei $\mathrm{x}$ giebt. Und noch eine eigenthitmliche Erscheinung tritt hier dentlich zu Tage, dass nämlich jede vierte Zwischenleiste breiter ist, als die übrigen alle, und jede zweite $Z$ wischenleiste etwas breiter als die erste und dritte. Auch die eingelagerten Körnchen sind in den breiteren Zwischenleisten grösser. In ihrer ganz typischen Anordnung erscheinen die Leisten in der Figur links oben.

Noch deutlicher sind dieselben Verhältnisse, welche mir üb. rigens nach anderen Methoden nicht aufgefallen sind, in Fig. 2 sichtbar. Diese ist ein anderes Stückchen contractiler Rinde von aussen gesehen und mit von innen anhaftendem Muskelmark, etwas stärker gequetscht, wodurch die faserigen, resistenteren Bestandtheile des Markes mit ihrer feingekörnten Umhüllung sich nach vorne und seitwärts umgeschlagen haben und hervorgedrängt worden sind. Die Verbindungen der in das Wabenwerk des Muskelmarkes wm eingebetteten Fibrillen mit den Zwischenleisten zl durch feine Fibrillen rf und lf sind sehr deutlich. In ihrer natürlichen Lage besitzen sowohl die Fibrillen rf; als auch jene mit If bezeichneten, welche als eine longitudinale Fortsetzung dcr Zwischenleisten erscheinen, eine radiüre Richtung zur Rinde, nur sind erstere im Präparat nach der Seite, letztere nach vorne umgebogen. Jede seitswärts umgebogene Fibrille rf begiebt sich zu einem scheinbaren Körnchen in einer Zwischenleiste, besonders deutlich zu sehen auf der rechten Seite der Figur, wo die Bruchlinie des Rindenstückes beinahe vollkommen einer Zwischenleiste entspricht. Diese Körnchen, welche in ziemlich regelmässigen Abständen hintereinander gereiht sind, entsprechen der Umbiegungsstelle resp. dem optischen Querschnitt der Fibrillen if, die sich, wie es besonders in Fig. 6, 7 und 9 deutlich zu sehen ist, als radiäre Mittel- oder Zwischenfibrillen in die Zwischenleisten fortsetzen. Da sich weiter in jeder Zwischenleiste auch longitudinale Mittelfibrillen befinden, das heisst die in radiärer Richtung in die Leiste eingetretenen Fibrillen sich in longitudinaler Richtung umbiegen, so können die Körnchen auch Kreuzungs- und Verlöthungspunkte von radiären und longitudinalen Mittelfibrillen darstellen. (Vergleiche den Längsschnitt der Rinde in Fig. 8 und die Querschnitte besonders in Fig. 6 und 7.) Die Verlöthung derselben wird durch die Perifibrillärsubstanz (Axoplasmaumhüllung der Nervenfibrillen nach anderen Autoren, u. A. Schiefferdecker) bewirkt; je nach- 
dem sie spärlicher oder reichlicher auftritt, erscheinen die Körnchen (ein Theil der sogenannten Varicositäten der leitenden Fibrille) kleiner oder grösser.

Das in dieser Figur abgebildete Stückchen Muskelrinde lag nicht ganz eben im Präparat; die mit $\mathbf{J}$ bezeichneten dunkleren Partien zeigen, bei scharfer Einstellung der Körnchen in der Partie A etwas unter der Aussenfläche der Rinde, die Imnenfläche der letzteren, wo sie an das Mark grenzt. An der vorderen Risslinie des Stückes sieht man eine Fibrille qf, welche quer zur Längsachse der Muskelfaser, hart an der Grenze von Mark und Rinde verläuft; sie steht einerseits mit den Fibrillen If, andererseits mit den mit Muskelplasma umhüllten Fibrillen des Markes in Verbindung. Bei $\alpha$ ist eine solche Verbindung mit einer Zwischenleiste und mit einer Radiärfibrille $\alpha_{1}$ durch Aendern der Einstellung genau verfolgt und eingezeichnet.

In den in Fig. 1 und 2 abgebildeten Quetschpräparaten sind die Fibrillen, welche in den folgenden Figuren mit nfi (Nervenfibrillen) bezeichnet wurden, durch ihre Farbe von dem Muskelplasma und der Grundsubstanz der Zwischenleisten wenig oder nicht differenzirt, sie sind nicht so scharf wie in meinen vergoldeten Schnittpräparaten gezeichnet und es haften ihnen feine Körnchen und Fädchen an, welche zu dem eigentlichen Muskelplasma gehören. Dass sie präformirt im Objekt vorhanden sind, zeigt ihre grosse Resistenz beim Quetşehen gegenüber dem eigentlichen Muskelplasma, welches durch die Einwirkung der schwachen Ameisensäurelösung (1:100) sehr weich wurde und hier kein festes, derbfaseriges Coagulum bildet, sondern sich leicht zerdrücken lässt. Dass aber die Fibrillen der Fig. 2 den leitenden Fibrillen der übrigen Figuren entsprechen, zeigt ihre Anordnung und ihre Verbindungen.

In den vergoldeten Schnitten dagegen, welche nach der auf Seite 348 und 349 meiner öfters citirten Ascarisarbeit beschriebenen Methode hergestellt sind, differenziren sich die leitenden Fibrillen, wie ich es wiederholt betont habe, sowohl durch eine sehr scharfe Zeichnung als anch durch eine sehr dunkel violette, beinahe schwarze Fürbung.

Das Muskelmark bekommt sie entweder vom Markbeutel her oder von besonderen Bündeln, resp. dicken Fibrillen, welche sich direkt zur Innenseite (Markseite) der Muskelfaser begeben (s. As- 
carisarbeit p. 342-343). Letzteres Verhalten ist in Fig. 3 dargestellt. Die Muskelfaser, in welche die dicke Fibrille $\mathrm{nf}_{1}$, umhüllt von einer Lamelle der in mehrere Blätter gespaltenen Interstitialmembran im (Ascarisarbeit p. 343-344), eintritt, liegt in der Nähe der ventralen Medianlinie. (Bei Q. ist der schräge Querschnitt eines dicken Markbeutelfortsatzes angedeutet, dessen Vereinigung mit der Medianlinie in einem weiteren Schnitt der Serie stattfindet.) Die contractile Rinde er ist bloss angedeutet. Bei $a, b$ und $\mathrm{c}$ sind an verschiedenen Stellen angetroffene Formen der contractilen Leisten halbschematisch eingezeichnet. Das Hauptgewicht wurde auf die genaue Einzeichnung sämmtlicher Verästelungen der Fibrille $\mathrm{nf}_{1}$ gelegt, welche sich in diesem $2 \mu$ dicken Schnitt der Serie befinden. Das verbindende Stück zwischen den Stellen $\alpha$ und $\beta$ liegt in dem nächstbenachbarten Schnitt. Ebenso lassen sich die Fibrillenstücke vnfi, als zu den Verzweigungen der Fibrille nfi $_{1}$ gehörend nachweisen. Die Fibrillenstücke vnfi $_{2}$ stammen anderswoher, nicht von der Fibrille $\mathrm{nf}_{1}$. Die hier angedenteten radiären Mittellinien rf sind nicht mit dem Zeichenapparat, sondern nachträglich eingetragen.

Figur 4, eine Uebergangsstelle der contractilen Rinde in die Markbeutelwand, zeigt den anderen Modus des Eintrittes der leitenden Fibrillen in das Muskelmark. Die Markbeutelwand zeigt zwischen zwei Lamellen der Interstitialmembran im den Durchschnitt eines Interstitialmuskels imu, in welchem längliche, dunkle Gebilde sichtbar sind, deren Natur ich nicht genau feststellen konnte (s. Ascarisarbeit p. 344-345). Zwei leitende Fibrillen verlaufen hier nebeneinander in der etwas schräg (tangential) getroffenen Beutelwand, von welchen besonders die dickere nfi, mit Ausnahme von einigen kleinen Strecken, an welchen sie ais benachbarten Schnitten ergänzt werden muss, innerhalb der Schnittdicke $(2-3 \mu)$ deutlich zu verfolgen ist. Man sieht, wie sich radiäre Aeste von ihr in die contractile Rinde begeben, welche hier übrigens zum grösseren Theil bloss angedeutet ist.

Auf die Frage, woher die Markbeutel ihre leitenden Fibrillen bekommen, giebt Fig. 5 Antwort, in welcher drei Querschnitte der sogenannten Markbeutelfortsätze oder „Muskelquerfortsätze“ in verschiedener Entfernung vom Markbeutel abgebildet sind. Sie sind von Lamellen der Interstitialmembran umhüllt, welche die äusserste Schichte der Wand der Markbeutelfortsätze ebenso 
wie der Markbeutel selbst bildet. In die Interstitialmembran sind hier Fibrillen von Interstitialmuskeln imu eingelagert, welche, mit einander anastomosirend und verschmelzend, ihrerseits ebenfalls eine gefensterte Membran darstellen. Bei a ist der Markbeutelfortsatz nicht weit von der Stelle getroffen, wo er in den Markbeutel übergeht. Die quer durchschnittenen leitenden Fibrillen erscheinen, je nach ihrer Stärke, als kleinere oder grössere, sehr dunkle, beinahe schwarze Punkte, welche sich hier besonders an einer Seite angesammelt haben. Von den Fibrillen dieses Fortsatzes setzt sich eine dickere Nervenfibrille nfi zusammen, welche gerade in der Schnittebene abbiegt und so einen längsgetroffenen Seitenast des Querfortsatzes bildet. Auch die Fibrillen, welche in den Seitenast einlenken, befinden sich zum Theil in der Schnittdicke und einige von ibnen sind ebenfalls längs getroffen. - Je näher man zum Markbeutel kommt, umsomehr peripherisch lagern sich die Fibrillen im Fortsatz, letzterer nähert sich also umsomehr dem röhrenförmigen Nerventypus; je weiter man ihn dagegen vom Markbeutel weg verfolgt, umso ähnlicher wird er dem anderen Nerventypus, den ich den bündelförmigen genannt habe. In solchen mehr und mehr ver jüngten Partien des Markbeutelfortsatzes, wie eine in dieser Figur bei b dargestellt ist, spielt der von Seiten des Markbeutels in ihn hineingesandte protoplasmatisch-saftige Theil eine immer geringere Rolle; die leitenden Primitivfibrillen dagegen, ebenso wie in den gleichfalls nach dem bündelförmigen Typus geformten Längsnerven des Ascariskörpers, eine immer grössere. Noch weiter können die einzelnen leitenden Fibrillen des noch mehr verjüngten Fortsatzes zu e in er dicken, sehr stark tingirten Fibrille verschmelzen, wie z. B. bei c, wo die äusserst starke Fibrille von einer geschichteten Zone perifibrillärer Substanz und der Interstitialmembran umhüllt wird. Die letztere Beschaffenheit besitzt der Markbeutelfortsatz sehr oft, als er in die betreffende Medianlinie, resp. in den.dort verlaufenden Längsnerv, oder sogar in den Schlundring selbst eintritt. Der Eintritt des so beschaffenen Fortsatzes in den betreffenden Mediannerv ist bei $\mathrm{R} 0 \mathrm{hde}$ in seiner ausführlichen Ascarisarbeit ${ }^{1}$ ) auf S. 90 geschildert. Die dicke Fibrille kann schräg durchgeschnitten, wie R o h d e sagt, in Form eines Zapfens in den "Axen-

1) Rohde, E., Muskel und Nerv. I. Ascaris. Zoologische Beiträge, Bd. III, Heft 2, 1892. 
cylinder" des Nerven hineinragen. In der That ändert sie aber an solchen Stellen bloss ihre Richtung und zerfällt bald in einzelne dünnere leitende Fibrillen, welche sich mit dem Längsnerv ununterbrochen in den Schlundring, gelegentlich aber bereits früher zu einzelnen Ganglienzellen, die in die Medianlinien eingestreut sind, begeben. Nach $\mathrm{R}$ oh de zerlegen sich die Zapfen „durch weiter fortgesetzte Spaltung in immer kleinere Stücke, bis schliesslich ihr 'Spongioplasma sich in einzelne Fibrillen aufgefasert hat, welche von denen des Axencylinders nicht mehr zu unterscheiden sind, so dass ein direkter Uebergang des Spongioplasmas der Muskelzelle in dasjenige des Axencylinders wahrscheinlich ist." Hätte Rohde das Muskelplasma von den dasselbe durchziehenden leitenden Primitivfibrillen zu unterscheiden gewusst, so hätte er diese Beobachtung gewiss ganz anders verwerthet.

Wenden wir uns nun aber zu den weiteren Figuren, welche sämmtlich die Vertheilung der leitenden Fibrillen in den Muskelfasern, bezüglicherweise ihren Uebergang in die Subcuticula veranschaulichen.

In Fig. 6 sind die genauen Querschnitte von zwei Muskelzellen dargestellt; von ihren Nachbarn ist die angrenzende contractile Rinde bloss angedeutet, nur rechts unten sind drei contractile Leisten auch eingezeichnet, weil an dieser Stelle zufällig ein direkter Uebergang einer radiären Mittelfibrille nfi in die benachbarte Muskelfaser deutlich zu sehen war. Einen solchen nicht häufig zu nennenden Uebergang sieht man übrigens auch in Fig. 10.

Von den zwei Muskelquerschnitten der Fig. 6 ist nur in dem rechten alles, was in dem Schnitte von $2 \mu$ Dicke vorhanden, das heisst bei dieser Vergoldung, welche mehr Einzelheiten als alle anderen Tinktionen enthüllt, und bei starker Beleuchtung in stark brechendem Einschlussmedium sichtbar gewesen ist, eingezeichnet. Links ist dagegen bloss der peripherische Theil, die Kante der betreffenden Muskelfaser gezeichnet; die contractilen Leisten sind bloss zum Theil und bei versehiedener Beleuchtung eingetragen: alle sind in umgekehrter Richtung, als die der rechten Muskelfaser beleuchtet, und zwar die seitlichen bei etwa s schräger, die mittleren und unteren bei ebenfalls schräger, aber mit der Leistendicke paralleler Beleuchtung. Das eigentliche Muskelmark ist auf dieser Seite überhaupt nicht gezeichnet, von den 
leitenden Primitivfibrillen bloss zwei stärkere mit ibren sämmtlichen in dem Schnitte befindlichen Verzweigungen. In die in der Figur vorhandene Zone der Subcuticula sc habe ich alle leit e nden Fibrillen eingezeichnet; die übrigen, bedeutend blasseren, gar nicht scharfen Fibrillen, bezüglicherweise so aussehenden Bestandtheile sind nur ganz wenig angedeutet; sie waren mit dem Zeichenapparat auch nicht sicher zu verfolgen.

Umso genauer konnten verfolgt werden - und wurden es auch - die Uebergänge der radiären Mittelfibrillen in die Subcuticula und in Subcuticularfasern; die Vereinigung mit den letzteren wurde nur insofern eingezeichnet, als sie in dem hier gezeichneten Schnitte selbst stattfindet. Aus demselben Grunde fehlt in der Zeichnung das Ergänzungsstück der starken Fibrille links bei x, und die 5 kleinen Stücklein bei y, wo die betreffende Fibrille einen kurzwelligen Verlauf, mit senkrecht auf die Schnittebene stehenden Wellen, besass. Ebenfalls ein solcher welliger Verlauf der Fibrille, jedoch noch ganz innerhalb der Schnittdicke, verursacht ihr stellenweise perlschnurartiges Aussehen in der rechten Muskelfaser, z. B. bei z. Ein anderes oben bereits erwähntes moniliformes Aussehen besitzen die feineren Verzweigungen der leitenden Fibrillen, z. B. bei $v$, und namentlich alle radiären Fortsätze, welche sie als radiäre Mittel- oder Zwischenfibrillen rmfi (nfi) in die contractile Rinde senden. Zwei solche Fortsätze in einer Ebene in einer Zwischenleiste der Rinde habe ich nur sehr selten gesehen und glaube, dass dieses normalerweise gar nicht vorkommt. Wie sie nun an den Kanten der Muskelfaser in die Subcuticula hinaustreten und sich dort, um dickere Subeuticularfibrillen zu bilden, sammeln, ist in der Figur unten deutlich zu sehen.

Obwohl in Fig. 6 rechts auch vom Muskelplasma, von dem eigentlichen Muskelmark alles Sichtbare eingezeichnet ist, so halte ich Fig. 9 doch nicht für überflüssig, da hier die Beschaffenheit des Muskelplasmas noch deutlicher hervortritt. Die contractile Rinde, welche diese Stelle des Markes rechts und links begrenzt, ist bloss angedeutet. Man sieht den grossen Gegensatz, in welchem die Wabenwände des Muskelplasmas, die Plasmazüge, zu den leitenden Fibrillen, die von ihnen umhüllt werden, stehen. Die schwarzen Punkte sind Querschnitte von leitenden Fibrillen. Auch hier ist es deutlich, dass bloss die dünneren Fibrillen, namentlich wo sie Aeste abgeben oder mit ans anderer Richtung kommen- 
den Fibrillen verlöthet werden, varieös sind. Wo an Kreuzungrspunkten keine Verdickung gezeichnet ist, liefen sie übereinander, ohne verlöthet zu sein, hinweg. Der Grenze der contractilen Rinde und des Markes folgt beiderseits je eine Fibrille.

Bloss durch solche, sich jederseits an der Grenze der contractilen Rinde haltenden Primitivfibrillen und ihren radiären Aesten ist das leitende Element in dem Querschnitt von Fig. 7 repräsentirt. Es ist der Querschnitt einer von ihren Nachbarn, welche hier gar nicht angedeutet sind, seitlich stark abgeplatteten Muskelzelle, unweit hinter dem Schlundring, $1002 \mu$ hinter dem Vorderende des Körpers (eines mittelgrossen $t$ von Asc. lumbricoides). Oben in der Figur tritt an Stelle der aufhörenden contractilen Rinde die Markbeutelwand ( $m a b=$ Markbeutel). Bei nfig sind sich umbiegende, resp. Seitenäste abgebende dickere Nervenfibrillen quer getroffen. Auch die hier an der Grenze zwischen Rinde und Mark verlaufenden Fibrillen (nfi $a_{1}$ und nfi $b_{1}$ ) sind Seitenäste von solchen. Die contractilen Leisten sind bloss in der unteren Hälfte der Figur, aber bier ganz genau in ihren Lagebeziehungen zn den radiären Aesten der zwei leitenden Fibrillen nfi $a_{1}$ und nfi $b_{1}$ eingetragen. Je eine von zwei Radiärfibrillen begrenzte Gruppe von contractilen Fibrillen efi entspricht einer contractilen Leiste, welche sich in erstere aufgelöst hat. Es sind hier verschiedene Uebergänge sichtbar zwischen der typischen radiären Leiste und den seheinbar regellos zerstreuten Gruppen von contractilen Primitivfibrillen. - Von den an der Aussenseite der Muskelfaser (in der Figur unten) heraustretenden leitenden Primitivfibrillen gehen zweie (nfi a) innerhalb des Schnittes $(2 \mu$ dick) verfolgbar in die betreffende Nachbarmuskelfaser über; eine (nf b) schmiegt sich der Aaussenseite des rechten Nachbarn an; zweie gehen in radiärer Richtung weiter in die Subeuticula hinein und verschmelzen dort, nach ziemlich langem, unabhängigen Verlauf mit anderen ähnlichen Fibrillen. Das Mark, in welchem hier das Granulöse überwiegt, im Gegensatz zu Fig. 6 und 9, wo das Alveoläre, Schaumige vorherrscht, ist bloss an zwei Stellen me gezeichnet. Auch die Interfibrillärmasse, die Grundsubstanz in den Zwischenleisten, sieht hier anders aus, indem in ihr feine blasse Körnchen und Fädchen deutlicher auftreten, offenbar jedoch bloss infolge der coagulirenden Wirkung des Reagens (heisser Sublimatalkohol). Die bisher besprochenen Abbildungen von Schnitten zeigten 
das Verhältniss der leitenden und des contractilen Elementes in den Muskelfasern im Querschnitt, wo sich ersteres innerhalb der Rinde in Form der radiären Mittelfibrillen hervorthut und bloss die Stellen als Verdickungen sehen lässt, an welchen sich eine radiäre Mittelfibrille in eine longitudinale umbiegt oder mit einer solchen sie kreuzenden verlöthet ist. Zur besseren Veranschaulichung der longitudinalen Mittelfibrillen habe ich hier in Fig. 8 die Abbildung eines in tangentialer (nach der F. E. S c h u l z e'schen Terminologie paratangentialer) Längsrichtung getroffenen Stückchens contractiler Rinde gewählt, und zwar einer so dünnen Lage, dass in ihr nicht einmal in jeder Zwischenleiste eine longitudinale Mittelfibrille enthalten ist, da die Abstände derselben hintereinander, besonders in diesem Körpertheil (etwa zwei

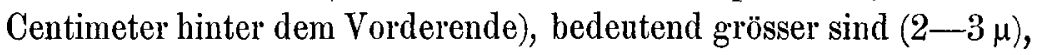
als die hier zur Untersuchung gekommene Lage von Rinde. au ist die, wegen der allmählichen Verjingung der Muskelfaser gegen ihre Enden zu, schräg getroffene äussere Grenze der contractilen Rinde. Die genau senkrecht auf der Schnittebene stehenden contractilen Leisten el reichten nach vorne nicht bis zur Grenzlinie an, wodurch ersichtlich, dass die contractilen Leisten nicht immer bis zur äussersten Oberfläche der Rinde hinausragen, sondern leztere eine dünne Aussenzone besitzen kann, die rein ans Interfibrillärsubstanz (die Grundsubstanz der Zwischenleisten ifs) besteht, welche keine wesentliche Struktur besitzt und als eingedichteter, eventuell mehr oder weniger veränderter, cuticularisirter, Zellsaft aufzufassen wäre. Die longitudinalen Mittelfibrillen nfi (l.m. fi) zeigen kleine Varices, welche ihren Kreuzungspunkten mit den radiären Mittelfibrillen entsprechen. Die in mehrere Lamellen gespaltene Interstitialsubstanz (im=Interstitialmembran, rechts eine solche, tangential getroffene, sich von ihrer Fläche präsentirend) schmiegt sich der Aussenzone der Rinde nicht unmittelbar an.

Dasselbe Verhalten ist auch in Fig. $10^{\circ}$ sichtbar, wo der Zwischenraum zwischen zwei Muskelfasern, von welchen bloss die contractile Rinde or angedeutet, und der Uebergang der Interstitialsubstanz in die Grundsubstanz der Subcuticula abgebildet ist. Zwischen Interstitialsubstanz und den entsprechenden Seitenflächen der Muskelfasern, zwischen welche sie sich hineinschiebt, existirt im Präparat beiderseits ein wahrscheinlich künstlich hervorgerufener Spaltraum; gerade dieser macht die Stelle besonders 
lehrreich. In der in dieser Weise eingekeilten Interstitialsubstanz sieht man die bereits stark verjüngten Enden zweier Muskelfasern hintereinander quer getroffen. Wie bekannt, bilden die Muskelfasern der Körperwand von Ascaris bloss eine Zellschichte, wo sie, seitlich stark abgeplattet, radiär nebeneinander stehen; nur ihre bereits stark verjüngten Enden können sich seitlich verschieben und so zu zweit, wie in Fig. 10, höchstens zu dreien hintereinander lagern. Bei ihrer Verjüngung schwindet aus ihnen das Markplasma immer mehr und schliesslich bestehen die Muskelenden bloss aus contractilen Leisten, beziehungsweise Fibrillen und körnig-fädig coagulirender Interfibrillärsubstanz. Wie die an den Aussenecken (Kanten) der Muskelfasern - je eine aus jeder Zwischenleiste in einem und demselben Niveau, nicht was hier, wohl aber in Fig. 6 deutlich ist - heraustretenden radiären Mittelfibrillen convergiren und so ein conisches Bündel, welehes zu einer dickeren Fibrille wird, bilden könmen, ist in der Figur links unten zu sehen: etwas Aehnliches hat, wie ich es auch in meiner vorigen Arbeit hervorgehoben habe, bereits $\mathrm{R}$ o b d e wahrgenommen, er hat es aber ziemlich ungenau gezeichnet (vergleiche seine Fig. 26 und 35 l. c., wo aus jedem Zwischenraum regellos mehrere Fädchen, in Fig. 26 rechts unten sogar pinselförmig. auseinanderstrahlend, heraustreten) und, als eingefleischter Hyaloplasmatiker, nach meiner Ueberzeugung wenigstens, 'ganz falsch gedeutet.

Und hiermit beende ich diesen Artikel in der Hoffnung das in meiner früheren Ascarisarbeit Auseinandergesetzte in ein schärferes Licht gestellt und.weiteren Missverständnissen vorgebeugt zu haben. Man möge es mir vorläufig verzeihen, wenn ich mich über verschiedene Punkte, welche ich bei einer anderen Gelegenheit besser erörtern zu können glaube, weiter "ausschweige". Ich weiss selbst am besten, wie einseitig meine Arbeit ist und wie viel in ihr noch fehlt, - sind ja darin nicht einmal die ethnographischen Verhältnisse der Somalineger behandelt, die doch auch glückliche Besitzer von Spulwürmern sein können.

Kolozsvár, im Februar 1894. 


\section{Erklärung der Abbildungen auf Tafel XXXVI.}

Sämmtliche Figuren sind mit dem Abbe'schen Zeichenapparat und - ausgenommen die schwächer vergrösserte Figur 1 - mit apochromatischen Oelimmersionsystemen von $Z$ eiss verfertigt, Fig. 1 u. 2 von Zupf- beziehungsweise Quetschpräparaten nach Goldchlorid-Ameisensururebehandlung des frischen lebenden Objectes, die übrigen Figuren von in Canadabalsam aufgehobenen Serienschnitten $(2-3 \mu$ dick) nach Fixirung mit heissem Sublimatalkohol und Vergoldung der am Objectträgrer aufgeklebten Serie, sämmtlich aus Ascaris lumbricoides.

Die Buchstaben bedeuten: $\mathrm{cr}=$ contractile Rinde, Rindenschicht der Muskelfaser; cl= contractile Leiste; $\mathrm{zl}=$ Zwischenleiste, Zwischenraum von je zwei contractilen Leisten; $w m=$ Wabenwerk des Muskelmarkes (Medulla), Muskelplasmazüge, welche die leitenden Fibrillen umhüllen; qf = quer, an der Grenze von Rinde und Mark verlaufende leitende Fibrillen; If = longitudinal umgebogene radiäre Mittelfibrille; $\mathrm{rf}=$ seitwärts umgebogene radiäre Mittelfibrille; $n f i=$ Nervenfibrillen (leitende Fibrillen); vnfi $=$ Verästelungen einer Nervenfibrille; nfiq od. $q \mathrm{~g} f=$ quergetroffene Nervenfibrillen; rf od. rmfi = radiäre Mitteloder Zwischenfibrille; lmf = longitudinale Mittel- oder Zwischenfibrille; me oder mep = Medullarplasma $;$ im = Interstitialmembran; imu = Interstitialmuskeln; is (im) = Interstitialsubstanz; sc $=$ Subcuticularschichte; seg $=$ Grundsubstanz der Subcuticula ; mab = Markbeutel; ifs = Interfibrillärsubstanz; em = Querschnitte von verjüngten Muskelfaserenden.

Fig. 1. Stück contractiler Muskelrinde von aussen gesehen; contractile Leisten vertical auf das Gesichtsfeld. Verlauf der Liniensysteme der Zwischenleisten. Vergr. 400 (vergl. p. 900, 901).

Fig. 2. Etwas gequetschtes Stück contractiler Rinde mit von unten anhaftendem Muskelmark. Die Körnchen und scheinbaren Körnchen der Zwischenleisten sämmtlich eingezeichnet. Vergr. 1500. Durch die starke Quellung erscheinen die contractilen Leisten zweimal so breit als im natürlichen Zustande (vergl. p. 901, 902).

Fig. 3. Eintritt einer dickeren leitenden Fibrille in die Muskelfaser und ihre Verzweigungen (meist quer getroffen, als Punkte sichtbar). Vergr. 1500 (vergl. p. 903).

Fig. 4. Uebergangsstelle von contractiler Rinde und Markbeutelwand. Vergr. 1500 (vergl. p. 903).

Fig. 5, a, b, c Querschnitte von Markbeutelfortsätzen; d der Fortsatz c längs getroffen. Vergr. 1500 (vergl. p. 903-904).

Fig. 6. Quersehnitt von zwei Muskelfasern (die rechte vollkommen ausgeführt) mit angrenzender Zone der Subcuticula. Links ist das eigentliche Mark nicht eingetragen. Vergr. 2000 (vergl, p. 905-907). 
Fig. 7. Querschnitt einer seitlich stärker abgeplatteten Muskelfaser, mit Auflösung der contractilen Leisten in Gruppen von einzelnen contractilen Primitivfibrillen. Vergr. 2000 (vergl. p. 907).

Fig. 8. Tangentialer Längsschnitt der Muskelrinde mit contractilen Leisten und longitudinalen Mittelfibrillen. Vergr. 1500 (vergI. p. 908).

Fig. 9. Das Muskelmark in ihrer schaumig-wabigen Beschaffenheit, mit eingebetteten sich verästelnden leitenden Primitivfibrillen. Vergr. 2000 (vergl. p. 906).

Fig. 10. Der keilförmige Zwischenraum von zwei Muskelfasern und der Subcuticularschicht, deren Grundsubstanz sich zwischen erstere hineinschiebt, mit zwei Querschnitten von stark verjünten Muskelfaserenden. Vergr. 1100 (vergl. p. 908-909).

\section{Ontogenetische Differenzirung des Ektoderms in Necturus.}

Von

Julia B. Platt.

I. Studie.

Hierzu Tafel XXXVII-XLII.

\section{Einleitung.}

Diese Arbeit wurde im Frühling 1893 in dem Laboratorium meines Freundes Dr. J. S. King sle y angefangen, der mir nicht nur die Ausriistung seines Laboratoriums zur Verfitgung stellte, sondern mir auch mit seiner praktischen Erfahrung und weitgehenden Belesenheit behilflich war. Fortgesetzt wurde die Arbeit in Woods Holl, wo die Marine Biological Society mir gütigst die Benützung eines Zimmers uiberliess; jetzt ist sie im Laboratorium des Hern Professors v. Kupffer in München vollendet und herausgegeben worden.

$\mathrm{Zu}$ dreifacher Dankbarkeit bin ich verpflichtet und mit besonderem Vergnügen gedenke ich der grossen Güte, mit welcher 


$$
1 \text { y }
$$
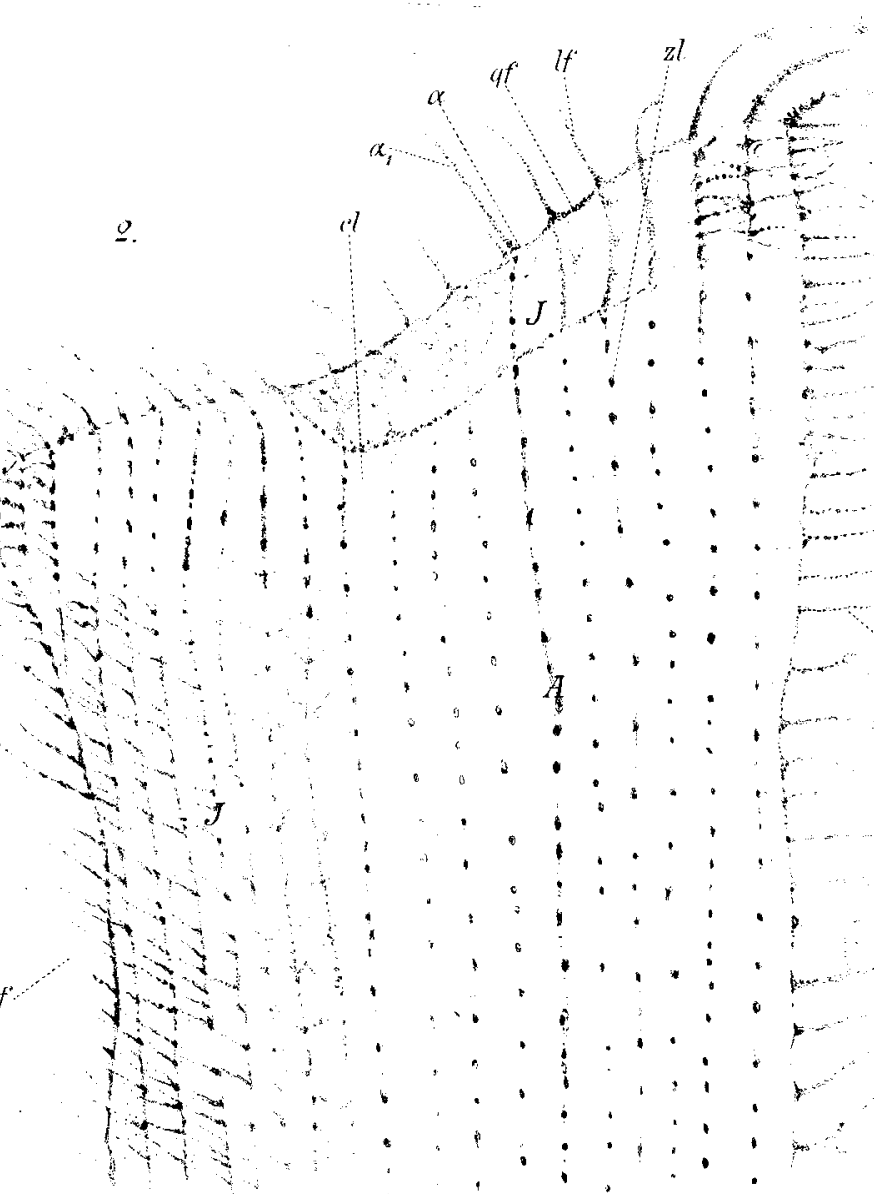
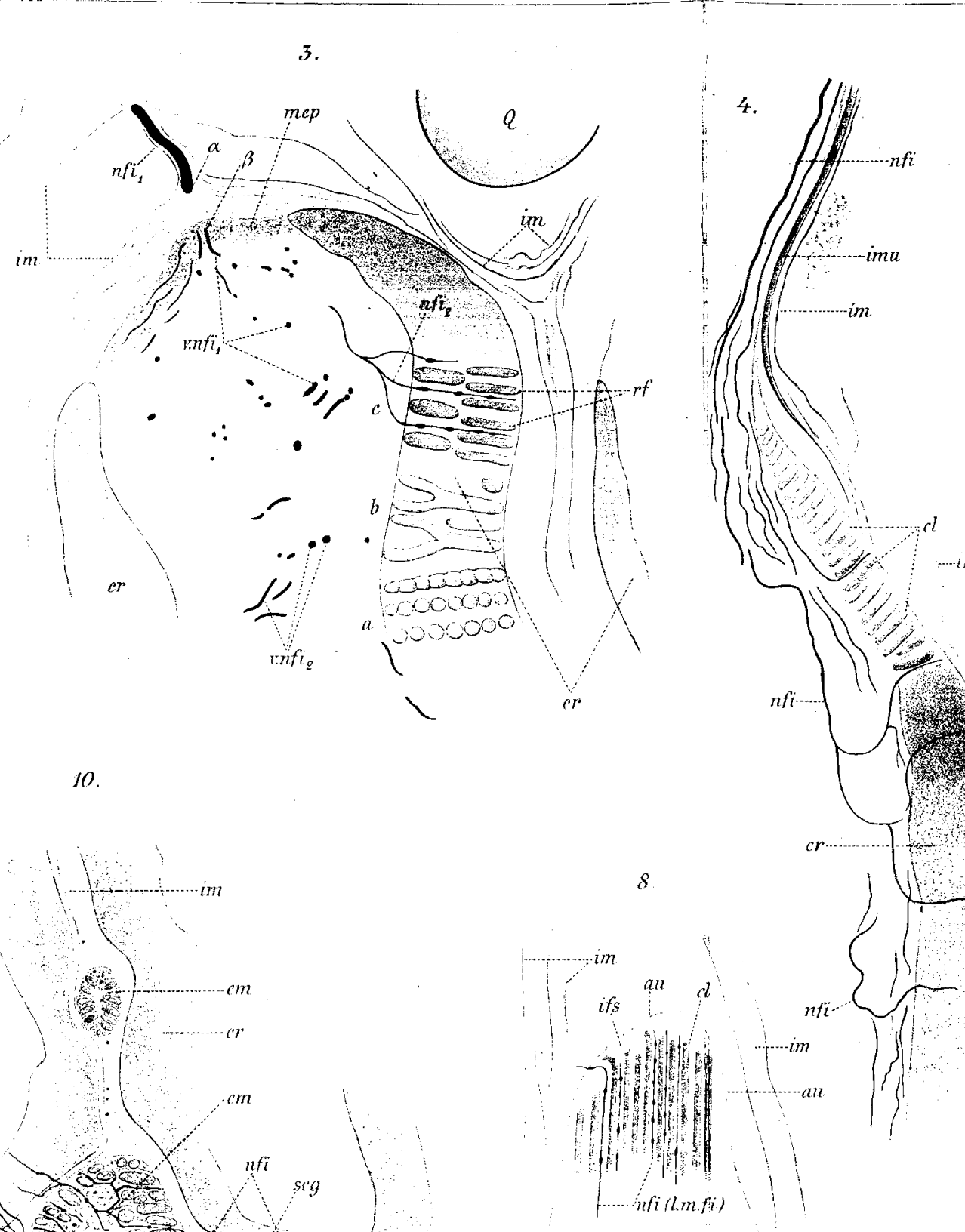
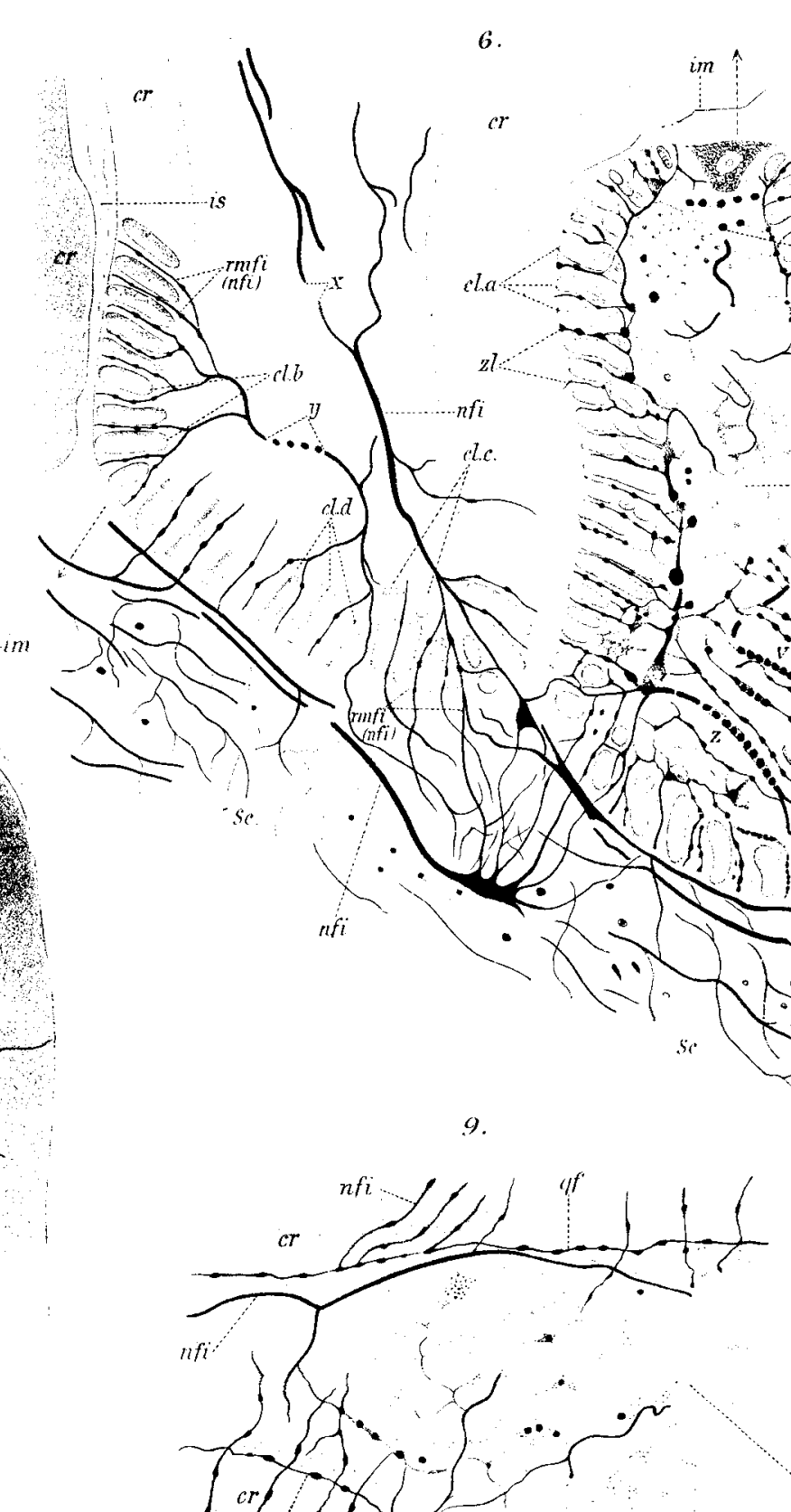
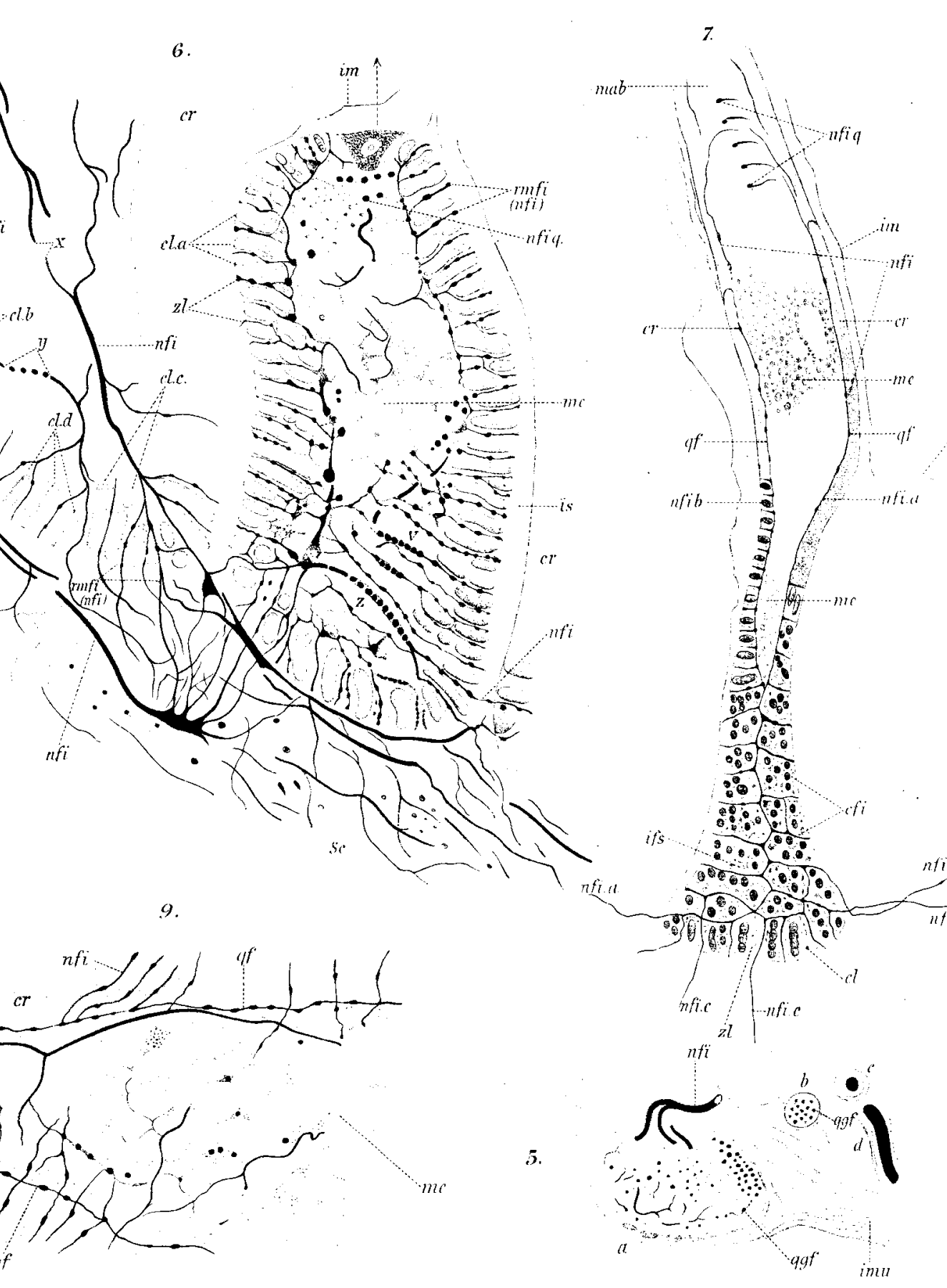